\title{
Multiple periodic solutions for damped vibration systems with superquadratic terms at infinity
}

\section{Ping $\mathrm{Li}^{1}$ and Guanwei Chen ${ }^{1 *}$ (B)}

"Correspondence: guanweic@163.com

${ }^{1}$ School of Mathematical Sciences, University of Jinan, Jinan, China

\begin{abstract}
By using variational methods, we obtain infinitely many nontrivial periodic solutions for a class of damped vibration systems with superquadratic terms at infinity. By using some weaker conditions, our results extend and improve some existing results in the literature. Besides, some examples are given to illustrate our results.
\end{abstract}

MSC: $34 \mathrm{C} 25 ; 70 \mathrm{H} 05$

Keywords: Damped vibration systems; Periodic solutions; Superquadratic

\section{Introduction and the main result}

In this paper, we study the existence of infinitely many nontrivial periodic solutions for the following damped vibration system:

$$
\left\{\begin{array}{l}
\ddot{u}+\left(q(t) I_{N \times N}+B\right) \dot{u}+\left(\frac{1}{2} B q(t)-A(t)\right) u+F_{u}(t, u)=0, \quad t \in \mathbb{R}, \\
u(0)-u(T)=\dot{u}(0)-\dot{u}(T)=0, \quad T>0,
\end{array}\right.
$$

where $u=u(t) \in C^{2}\left(\mathbb{R}, \mathbb{R}^{N}\right), I_{N \times N}$ is the $N \times N$ identity matrix, $q(t) \in L^{1}(\mathbb{R} ; \mathbb{R})$ is $T$ periodic and satisfies $\int_{0}^{T} q(t) d t=0, A(t)=\left[a_{i j}(t)\right]$ is a $T$-periodic symmetric $N \times N$ matrixvalued function with $a_{i j} \in L^{\infty}(\mathbb{R} ; \mathbb{R})(\forall i, j=1,2, \ldots, N), B=\left[b_{i j}\right]$ is an antisymmetric $N \times N$ constant matrix.

When $B=0$ (zero matrix), the authors [6] studied the special case of (1.1) and obtained the existence and multiplicity of periodic solutions. When $B \neq 0$, the author [2] obtained infinitely many periodic solutions of $(1.1)$ with $F(t, u)$ satisfying the asymptotically quadratic condition:

$$
\lim _{|u| \rightarrow \infty} \frac{F(t, u)}{|u|^{2}}=V(t) \quad \text { uniformly for } t \in[0, T] \text {, where } \inf _{t \in[0, T]} V(t)>0,
$$

(c) The Author(s) 2019. This article is licensed under a Creative Commons Attribution 4.0 International License, which permits use, sharing, adaptation, distribution and reproduction in any medium or format, as long as you give appropriate credit to the original author(s) and the source, provide a link to the Creative Commons licence, and indicate if changes were made. The images or other third party material in this article are included in the article's Creative Commons licence, unless indicated otherwise in a credit line to the material. If material is not included in the article's Creative Commons licence and your intended use is not permitted by statutory regulation or exceeds the permitted use, you will need to obtain permission directly from the copyright holder. To view a copy of this licence, visit http://creativecommons.org/licenses/by/4.0/. 
the authors [4] obtained one existence result and two multiplicity results with $F(t, u)$ satisfying the superquadratic condition:

$$
0<\mu F(t, u) \leq\left(F_{u}(t, u), u\right), \quad \forall t \in[0, T], \forall|u| \geq r
$$

where $\mu>2$ and $r \geq 0$ are some constants, $(\cdot, \cdot)$ and $|\cdot|$ denote the inner product and the associated norm in $\mathbb{R}^{\mathbb{N}}$; the author [3] used a more general superquadratic condition $\left(\lim _{|u| \rightarrow \infty} \frac{F(t, u)}{|u|^{2}}=+\infty\right.$ uniformly for $\left.t \in[0, T]\right)$ and obtained infinitely many periodic solutions for (1.1).

By the more general superquadratic condition used in [3] and some weaker conditions, we also obtain infinitely many periodic solutions for (1.1). Our main result reads as follows.

Theorem 1.1 System (1.1) has infinitely many nontrivial T-periodic solutions if $F(t, u)$ is $T$-periodic in $t$ and even in $u$, and the following conditions hold:

$\left(F_{1}\right) F(t, u)$ is measurable in $t$ for every $u \in \mathbb{R}^{\mathbb{N}}$ and continuously differentiable in $u$ for a.e. $t \in[0, T]$, and there exist $a \in C\left(\mathbb{R}^{+}, \mathbb{R}^{+}\right), b \in L^{1}\left([0, T] ; \mathbb{R}^{+}\right)$such that

$$
|F(t, u)| \leq a(|u|) b(t), \quad\left|F_{u}(t, u)\right| \leq a(|u|) b(t), \quad \forall(t, u) \in[0, T] \times \mathbb{R}^{N} .
$$

$\left(F_{2}\right) F(t, u) \geq 0, \forall(t, u) \in[0, T] \times \mathbb{R}^{N}$, and

$$
\lim _{|u| \rightarrow \infty} \frac{F(t, u)}{|u|^{2}}=+\infty \quad \text { uniformly for } t \in[0, T]
$$

$\left(F_{3}\right)$ There exists $\alpha>2$ such that

$$
\lim _{|u| \rightarrow \infty} \frac{F_{u}(t, u)}{|u|^{\alpha-1}}<+\infty \quad \text { uniformly for } t \in[0, T]
$$

$\left(F_{4}\right)$ There are constants $b>0$ and $1 \leq \beta \in(\alpha-2,+\infty)$ such that

$$
\lim _{|u| \rightarrow \infty} \inf \frac{\left(F_{u}(t, u), u\right)-2 F(t, u)}{|u|^{\beta}}>b \quad \text { uniformly for } t \in[0, T] .
$$

To compare our result with the most related result [3], we firstly describe the result in [3].

Theorem 1.2 ([3]) System (1.1) has infinitely many nontrivial T-periodic solutions if $F \in$ $C^{1}\left(\mathbb{R} \times \mathbb{R}^{N}, \mathbb{R}\right)$ is T-periodic in t and even in $u$, and it satisfies the following conditions:

$\left(S F_{1}\right) F(t, 0)=0, \forall t \in[0, T]$, and there are two constants $d_{1}>0$ and $\alpha_{1}>2$ such that

$$
\left|F_{u}(t, u)\right| \leq d_{1}\left(1+|u|^{\alpha_{1}-1}\right), \quad \forall(t, u) \in[0, T] \times \mathbb{R}^{N} .
$$

$\left(S F_{2}\right) \frac{1}{2}\left(F_{u}(t, u), u\right) \geq F(t, u) \geq 0$ for all $(t, u) \in[0, T] \times \mathbb{R}^{N}$ and

$$
\lim _{|u| \rightarrow \infty} \frac{F(t, u)}{|u|^{2}}=+\infty \quad \text { uniformly for } t \in[0, T]
$$


$\left(\mathrm{SF}_{3}\right)$ There is a constant $b>0$ such that

$$
\lim _{|u| \rightarrow \infty} \inf \frac{\left(F_{u}(t, u), u\right)-2 F(t, u)}{|u|^{\alpha_{1}}}>b \quad \text { uniformly for } t \in[0, T] .
$$

Remark 1.1 The method of Theorem 1.1 is based on the fountain theorem of Bartsch [1], which is essentially different from the variant fountain theorem developed by Zou [7] used in [3]. Our result extends and improves the result in [3]. The reasons are as follows:

(1) We only need $F$ to satisfy $\left(F_{1}\right)$ rather than $F \in C^{1}\left(\mathbb{R} \times \mathbb{R}^{N}, \mathbb{R}\right)$.

(2) We remove the condition $\frac{1}{2}\left(F_{u}(t, u), u\right) \geq F(t, u)$ for all $(t, u) \in[0, T] \times \mathbb{R}^{N}$ in $\left(S F_{2}\right)$.

(3) Condition $\left(F_{3}\right)$ is weaker than $\left(S F_{1}\right)$. Indeed, condition $\left(S F_{1}\right)$ implies

$$
|F(t, u)-F(t, 0)|=\left|\int_{0}^{1}\left(F_{u}(t, s u), u\right) d s\right| \leq d_{1}\left(|u|+|u|^{\alpha_{1}}\right), \quad \forall(t, u) \in[0, T] \times \mathbb{R}^{N},
$$

it follows from the continuity of $F(\cdot, 0)$ that (for all $\alpha \geq \alpha_{1}$ )

$$
\begin{aligned}
& \lim _{|u| \rightarrow \infty} \sup \frac{|F(t, u)|}{|u|^{\alpha}} \\
& \quad \leq \lim _{|u| \rightarrow \infty} \sup \frac{d_{1}\left(|u|+|u|^{\alpha_{1}}\right)+\max _{t \in[0, T]}|F(t, 0)|}{|u|^{\alpha}}<+\infty \quad \text { uniformly for } t \in[0, T] .
\end{aligned}
$$

(4) The constant $\beta$ in our condition $\left(F_{4}\right)$ is more general than $\alpha_{1}$ in $\left(S F_{3}\right)$.

Example 1.1 The following example is given to illustrate our result. Let

$$
F(t, u):=h(t)|u|^{2} \ln \left(1+|u|^{2}\right), \quad u \in \mathbb{R}^{N}, t \in[0, T],
$$

where $h \in L^{\infty}\left(0, T ; \mathbb{R}^{+}\right)$with $\inf _{t \in[0, T]} h(t)>0$. Then

$$
F_{u}(t, u)=2 h(t) \ln \left(1+|u|^{2}\right) u+\frac{2 h(t)|u|^{2} u}{1+|u|^{2}} .
$$

It is not hard to check that the function satisfies our conditions $\left(F_{1}\right)-\left(F_{4}\right)$.

The rest of our paper is organized as follows. In Sect. 2, we establish variational framework associated with (1.1) and give some preliminary lemmas, which are useful in the proof of our result, and then we give the detailed proof of our main result.

\section{Variational frameworks and the proof of Theorem 1.1}

Let $\|\cdot\|_{p}$ denote the norm of $L^{p}\left([0, T] ; \mathbb{R}^{N}\right)$ for any $p \in[1, \infty]$. Let

$$
\begin{aligned}
W:= & \left\{u=u(t):[0, T] \rightarrow \mathbb{R}^{N} \mid u \text { is absolutely continuous, } u(0)=u(T),\right. \text { and } \\
& \left.\dot{u} \in L^{2}\left([0, T] ; \mathbb{R}^{N}\right)\right\}
\end{aligned}
$$

with the inner product

$$
(u, v)_{W}:=\int_{0}^{T}[(u, v)+(\dot{u}, \dot{v})] d t, \quad \forall u, v \in W .
$$

The corresponding norm is defined by $\|u\|_{W}=(u, u)_{W}^{1 / 2}$. Obviously, $W$ is a Hilbert space. 
Let

$$
Q(t):=\int_{0}^{t} q(s) d s
$$

and

$$
\|u\|_{0}:=\left(\int_{0}^{T} e^{Q(t)}\left(|u|^{2}+|\dot{u}|^{2}\right) d t\right)^{1 / 2}, \quad u \in W
$$

Obviously, the norm $\|\cdot\|_{0}$ is equivalent to the usual one $\|\cdot\|_{W}$ on $W$. We denote by $\langle\cdot, \cdot\rangle_{0}$ the inner product corresponding to $\|\cdot\|_{0}$ on $W$.

The corresponding functional for problem (1.1) is defined by

$$
\Phi(u):=\frac{1}{2} \int_{0}^{T} e^{Q(t)}\left[|\dot{u}|^{2}+(B u, \dot{u})+(A(t) u, u)\right] d t-\int_{0}^{T} e^{Q(t)} F(t, u) d t, \quad u \in W .
$$

Let $L: W \rightarrow W^{*}\left(W^{*}\right.$ is the dual space of $\left.W\right)$ be an operator defined by

$$
L u(v):=\int_{0}^{T} e^{Q(t)}\left[(B \dot{u}, v)+\frac{1}{2} q(t)(B u, v)\right] d t, \quad \forall u, v \in W .
$$

We can identify $W^{*}$ with $W$ by Riesz representation theorem, so $L u$ can be viewed as a function belonging to $W$ such that

$$
L u(v)=\langle L u, v\rangle_{0}, \quad \forall u, v \in W .
$$

It is not hard to check that $L$ is a bounded linear operator on $W$. From the discussion in [4], we get that $L$ is self-adjoint and compact on $W$. Since $B$ is an antisymmetric $N \times N$ constant matrix, it follows from integration by parts that

$$
\langle L u, u\rangle_{0}=\int_{0}^{T} e^{Q(t)}\left[(B \dot{u}, u)+\frac{1}{2} q(t)(B u, u)\right] d t=\int_{0}^{T} e^{Q(t)}[(B \dot{u}, u)] d t .
$$

We define an operator $K: W \rightarrow W^{*}$ by

$$
\langle K u, v\rangle_{0}=\langle L u, v\rangle_{0}+\int_{0}^{T} e^{Q(t)}\left(\left(I_{N \times N}-A(t)\right) u, v\right) d t, \quad \forall u, v \in W .
$$

Then it is easy to check that $K$ is a bounded self-adjoint linear operator. Therefore, the definitions of $\langle\cdot, \cdot\rangle_{0}$ and $K$ imply that $\Phi$ defined in (2.1) can be rewritten as

$$
\Phi(u)=\frac{1}{2}\langle(I-K) u, u\rangle_{0}-\int_{0}^{T} e^{Q(t)} F(t, u) d t, \quad u \in W,
$$

where $I$ denotes the identity operator.

By the classical spectral theory, we have the decomposition

$$
W=W^{0} \oplus W^{-} \oplus W^{+},
$$


where $W^{0}:=\operatorname{ker}(I-K), W^{+}$and $W^{-}$are the positive and negative spectral subspaces of $I-K$ in $W$, respectively. Besides, $W^{-}$and $W^{0}$ are finite dimensional (see [4]). Obviously, we can define a new equivalent inner product $\langle\cdot, \cdot\rangle$ on $W$ with corresponding norm $\|\cdot\|$ such that

$$
\langle(I-K) u, u\rangle_{0}= \pm\|u\|^{2}, \quad \forall u \in W^{ \pm}
$$

Then we have

$$
\begin{aligned}
\Phi(u) & =\frac{1}{2}\langle(I-K) u, u\rangle_{0}-I(u) \\
& =\frac{1}{2}\left(\left\|u^{+}\right\|^{2}-\left\|u^{-}\right\|^{2}\right)-I(u), \quad u \in W,
\end{aligned}
$$

where $I(u):=\int_{0}^{T} e^{Q(t)} F(t, u) d t$. Then, by the assumptions of $F$, we know that $I$ and $\Phi$ are continuously differentiable and

$$
\Phi^{\prime}(u) v=\left\langle u^{+}, v^{+}\right\rangle-\left\langle u^{-}, v^{-}\right\rangle-I^{\prime}(u) v, \quad I^{\prime}(u) v=\int_{0}^{T} e^{Q(t)}\left(F_{u}(t, u), v\right) d t
$$

where $u, v \in W=W^{-} \oplus W^{0} \oplus W^{+}$with $u=u^{-}+u^{0}+u^{+}$and $v=v^{-}+v^{0}+v^{+}$; besides, the $T$-periodic solutions of (1.1) are the critical points of the $C^{1}$ functional $\Phi: W \rightarrow \mathbb{R}$ (see [4]).

Since the embedding of $W$ into $C\left(0, T ; \mathbb{R}^{N}\right)$ is compact, there exists a constant $C>0$ such that

$$
\|u\|_{\infty} \leq C\|u\|, \quad \forall u \in W
$$

where $\|u\|_{\infty}=\max _{t \in[0, T]}|u(t)|$. Besides, by the Sobolev embedding theorem, we get directly the following lemma.

Lemma 2.1 $W$ is compactly embedded in $L^{p}\left([0, T] ; \mathbb{R}^{N}\right), \forall p \in[1,+\infty]$.

In order to prove Theorem 1.1, we state the fountain theorem of Bartsch (see $[1,5])$. Let W be a Banach space with the norm $\|\cdot\|$ and $W:=\overline{\bigoplus_{m \in \mathbb{N}} X_{m}}$ with $\operatorname{dim} X_{m}<\infty$ for any $m \in \mathbb{N}$. Set

$$
Y_{k}:=\bigoplus_{m=1}^{k} X_{m}, \quad Z_{k}:=\overline{\bigoplus_{m=k}^{\infty} X_{m}} .
$$

Lemma 2.2 (Fountain theorem) We assume that $\Phi \in C^{1}(X, \mathbb{R})$ satisfies the Cerami condition, $\Phi(-u)=\Phi(u)$. For almost every $k \in N$, there exist $\rho_{k}>r_{k}>0$ such that

(i) $a_{k}:=\inf _{u \in Z_{k},\|u\|=r_{k}} \Phi(u) \rightarrow+\infty$ as $k \rightarrow \infty$.

(ii) $b_{k}:=\max _{u \in Y_{k},\|u\|=\rho_{k}} \Phi(u) \leq 0$.

Then $\Phi$ has a sequence of critical values tending to $+\infty$.

Remark 2.1 Under the Palais-Smale (PS) condition, the fountain theorem is established in $[1,5]$. Because the deformation theorem holds true under the Cerami condition, we know 
the fountain theorem is still valid under the Cerami condition. Here, if any sequence $u_{n} \subset$ $X$ such that $\left\{\Phi\left(u_{n}\right)\right\}$ is bounded and $\left\|\Phi^{\prime}\left(u_{n}\right)\right\|\left(1+\left\|u_{n}\right\|\right) \rightarrow 0$ as $n \rightarrow \infty$ has a convergent sequence, we say that $\Phi \in C^{1}(X, R)$ satisfies the Cerami condition, such a subsequence is then called a Cerami sequence.

Lemma 2.3 If assumptions $\left(F_{1}\right),\left(F_{3}\right)$, and $\left(F_{4}\right)$ hold, then $\Phi$ satisfies the Cerami condition $(C)$.

Proof We assume that, for any sequence $\left\{u_{n}\right\} \subset W,\left\{\Phi\left(u_{n}\right)\right\}$ is bounded and $\left\|\Phi^{\prime}\left(u_{n}\right)\right\| \times$ $\left(1+\left\|u_{n}\right\|\right) \rightarrow 0$.

Part 1 . We firstly prove the boundedness of $\left\{u_{n}\right\}$. There is a constant $M>0$ such that

$$
\left|\Phi\left(u_{n}\right)\right| \leq M \quad \text { and } \quad\left\|\Phi^{\prime}\left(u_{n}\right)\right\|\left(1+\left\|u_{n}\right\|\right) \leq M, \quad \forall n \in \mathbb{N}^{+} .
$$

It follows from $\left(F_{4}\right)$ that there exist $c_{1}>0$ and $M_{1}>1$ such that

$$
\left(F_{u}(t, u), u\right)-2 F(t, u) \geq c_{1}|u|^{\beta}, \quad \forall|u| \geq M_{1}, \forall t \in[0, T] .
$$

By $\left(F_{1}\right)$, one has that

$$
\left|\left(F_{u}(t, u), u\right)-2 F(t, u)\right| \leq c_{2} b(t), \quad \forall|u| \leq M_{1}, \forall t \in[0, T]
$$

where $c_{2}=\left(M_{1}+2\right) \max _{s \in\left[0, M_{1}\right]} a(s)$. Combining (2.5) and (2.6), we get that

$$
\left(F_{u}(t, u), u\right)-2 F(t, u) \geq c_{1}|u|^{\beta}-c_{1} M_{1}^{\beta}-c_{2} b(t), \quad \forall|u| \in \mathbb{R}^{N}, \forall t \in[0, T] .
$$

It follows from $e^{Q(t)} \geq d_{1}$ for some constant $d_{1}>0(\forall t \in[0, T])$, (2.4), (2.7), the definitions of $\Phi(u)$ and $\Phi^{\prime}(u)$ that

$$
\begin{aligned}
3 M & \geq 2 \Phi\left(u_{n}\right)-\left\langle\Phi^{\prime}\left(u_{n}\right), u_{n}\right\rangle \\
& =\int_{0}^{T} e^{Q(t)}\left[\left(F_{u}\left(t, u_{n}\right), u_{n}\right)-2 F\left(t, u_{n}\right)\right] d t \\
& \geq d_{1} c_{1} \int_{0}^{T}\left|u_{n}\right|^{\beta} d t-d_{1} c_{1} M_{1}^{\beta} T-d_{1} c_{2} \int_{0}^{T} b(t) d t, \quad \forall n \in \mathbb{N}^{+} .
\end{aligned}
$$

By $(2.8)$ and $b \in L^{1}\left([0, T] ; \mathbb{R}^{+}\right)$, we have

$$
\int_{0}^{T}\left|u_{n}\right|^{\beta} d t \leq D, \quad \forall n \in \mathbb{N}^{+}
$$

for some $D>0$. Let $\Pi_{n}=\left\{t \in[0, T]:\left|u_{n}\right| \geq M_{1}\right\}$, then we have

$$
\int_{\Pi_{n}}\left|u_{n}\right|^{\beta} d t \leq D_{1}, \quad \forall n \in \mathbb{N}^{+}
$$

for some $D_{1}>0$. Since $\beta \geq 1$, we also have

$$
\int_{\Pi_{n}}\left|u_{n}\right| d t \leq D_{1}, \quad \forall n \in \mathbb{N}^{+} .
$$


For any $n \in N$, let $\chi_{n}: \mathbb{R} \rightarrow \mathbb{R}$ be the indicator of $\Pi_{n}$, that is,

$$
\chi_{n}(t):=\left\{\begin{array}{ll}
1, & t \in \Pi_{n}, \\
0, & t \notin \Pi_{n},
\end{array} \quad \forall n \in \mathbb{N}^{+} .\right.
$$

Then, by the definition of $\Pi_{n}$ and (2.10), we have

$$
\left\|\left(1-\chi_{n}\right) u_{n}\right\|_{\infty} \leq M_{1} \quad \text { and } \quad\left\|\chi_{n} u_{n}\right\|_{1} \leq D_{1}, \quad \forall n \in \mathbb{N}^{+} \text {. }
$$

It follows from the equivalence of any two norms on a finite-dimensional space $W^{0} \oplus W^{-}$ that

$$
\begin{aligned}
\left\|u_{n}^{-}\right\|_{2}^{2} & =\left(u_{n}^{-}, u_{n}\right)_{2} \\
& =\left(u_{n}^{-},\left(1-\chi_{n}\right) u_{n}\right)_{2}+\left(u_{n}^{-}, \chi_{n} u_{n}\right)_{2} \\
& \leq\left\|\left(1-\chi_{n}\right) u_{n}\right\|_{\infty} \cdot\left\|u_{n}^{-}\right\|_{1}+\left\|u_{n}^{-}\right\|_{\infty} \cdot\left\|\chi_{n} u_{n}\right\|_{1} \\
& \leq\left(h_{1}\left\|\left(1-\chi_{n}\right) u_{n}\right\|_{\infty}+h_{2}\left\|\chi_{n} u_{n}\right\|_{1}\right)\left\|u_{n}^{-}\right\|_{2} \\
& \leq\left(h_{1} M_{1}+h_{2} D_{1}\right)\left\|u_{n}^{-}\right\|_{2}, \quad \forall n \in \mathbb{N}^{+}
\end{aligned}
$$

for some $h_{1}, h_{2}>0$. Therefore,

$$
\left\|u_{n}^{-}\right\|_{2} \leq h_{1} M_{1}+h_{2} D_{1}, \quad \forall n \in \mathbb{N}^{+}
$$

which together with the equivalence of any two norms on a finite-dimensional space $W^{0} \oplus$ $W^{-}$implies that

$$
\left\|u_{n}^{-}\right\| \leq D_{2}, \quad \forall n \in \mathbb{N}^{+}
$$

for some $D_{2}>0$.

It follows from $\left(F_{3}\right)$ that there exist $c_{3}>0$ and $M_{2}>0$ such that

$$
F(t, u) \leq c_{3}|u|^{\alpha}, \quad \forall|u| \geq M_{2}, \forall t \in[0, T]
$$

By $\left(F_{1}\right)$, one has that

$$
|F(t, u)| \leq c_{4} b(t), \quad \forall|u| \leq M_{2}, \forall t \in[0, T],
$$

where $c_{4}=\max _{s \in\left[0, M_{2}\right]} a(s)$. Hence, we obtain

$$
F(t, u) \leq c_{3}|u|^{\alpha}+c_{4} b(t), \quad \forall|u| \in \mathbb{R}^{N}, \forall t \in[0, T]
$$

By (2.4), (2.11), (2.12), and $e^{Q(t)} \leq d_{2}$ for some constant $d_{2}>0(\forall t \in[0, T])$, we have

$$
\begin{aligned}
\frac{1}{2}\left\|u_{n}\right\|^{2} & =\Phi\left(u_{n}\right)+\left\|u_{n}^{-}\right\|+\int_{0}^{T} e^{Q(t)} F\left(t, u_{n}\right) d t \\
& \leq M+D_{2}+d_{2} c_{3} \int_{0}^{T}\left|u_{n}\right|^{\alpha} d t+d_{2} c_{4} \int_{0}^{T} b(t) d t, \quad \forall n \in \mathbb{N}^{+}
\end{aligned}
$$


If $\beta>\alpha$, Hölder's inequality and (2.9) imply that

$$
\int_{0}^{T}\left|u_{n}\right|^{\alpha} d t \leq T^{(\beta-\alpha) / \beta}\left(\int_{0}^{T}\left|u_{n}\right|^{\beta} d t\right)^{\alpha / \beta}<+\infty, \quad \forall n \in \mathbb{N}^{+} .
$$

It follows from (2.13), (2.14), and $b \in L^{1}\left([0, T] ; \mathbb{R}^{+}\right)$that $\left(u_{n}\right)$ is bounded.

If $\beta \leq \alpha$, using (2.2), we have that

$$
\begin{aligned}
\int_{0}^{T}\left|u_{n}\right|^{\alpha} d t & =\int_{0}^{T}\left|u_{n}\right|^{\beta}\left|u_{n}\right|^{\alpha-\beta} d t \\
& \leq\left\|u_{n}\right\|_{\infty}^{\alpha-\beta} \int_{0}^{T}\left|u_{n}\right|^{\beta} d t \\
& \leq C^{\alpha-\beta}\left\|u_{n}\right\|^{\alpha-\beta} \int_{0}^{T}\left|u_{n}\right|^{\beta} d t, \quad \forall n \in \mathbb{N}^{+} .
\end{aligned}
$$

Noting the fact that $\alpha-\beta<2$, it follows from (2.9), (2.13), and $b \in L^{1}\left([0, T] ; \mathbb{R}^{+}\right)$that $\left(u_{n}\right)$ is bounded.

Part 2. Then we prove that the sequence $\left\{u_{n}\right\}$ has a convergent sequence. The boundedness of $\left\{u_{n}\right\}$ implies that $u_{n} \rightarrow u$ in $W$. First, we prove

$$
\int_{0}^{T} e^{Q(t)}\left(F_{u}\left(t, u_{n}\right), u_{n}-u\right) d t \rightarrow 0, \quad n \rightarrow \infty
$$

Note that Lemma 2.1 implies that $u_{n} \rightarrow u$ in $L^{p}$ and there is a constant $c_{5}>0$, we have

$$
\left\|u_{n}-u\right\|_{p} \rightarrow 0, \quad\left\|u_{n}\right\|_{p} \leq c_{5}\|u\|, \quad \forall 1 \leq p<\infty
$$

If $|u| \geq M_{3}, M_{3}>0$, it follows from $\left(F_{3}\right)$ that there exists $c_{6}>0$ such that

$$
F_{u}(t, u) \leq c_{6}|u|^{\alpha-1}, \quad \forall|u| \geq M_{3}, \forall t \in[0, T] .
$$

According to the boundedness of $\left(u_{n}\right)$ and Lemma 2.1, we have $\left\|u_{n}\right\|<\infty$. It follows from $e^{Q(t)} \leq d_{2}$, (2.16), (2.17), and Hölder's inequality that

$$
\begin{aligned}
& \int_{0}^{T} e^{Q(t)}\left(F_{u}\left(t, u_{n}\right), u_{n}-u\right) d t \\
& \quad \leq d_{2} \int_{0}^{T}\left(c_{6}|u|^{\alpha-1}\right)\left(u_{n}-u\right) d t \\
& \quad \leq c_{6} d_{2}\left\|u_{n}-u\right\|_{\alpha} \cdot\left\|u_{n}\right\|_{\alpha}^{\alpha-1} \\
& \quad \leq c_{5}^{\alpha-1} c_{6} d_{2}\left\|u_{n}-u\right\|_{\alpha} \cdot\left\|u_{n}\right\|^{\alpha-1} \rightarrow 0 .
\end{aligned}
$$

If $|u| \leq M_{3}$, by $\left(F_{1}\right)$, one has that

$$
\left|F_{u}(t, u)\right| \leq c_{7} b(t), \quad \forall|u| \leq M_{3}, \forall t \in[0, T]
$$


where $c_{7}=\max _{s \in\left[0, M_{3}\right]} a(s)$. By Lemma $2.1,(2.19), e^{Q(t)} \leq d_{2}$, and $b \in L^{1}\left([0, T] ; \mathbb{R}^{+}\right)$, we obtain

$$
\begin{aligned}
& \int_{0}^{T} e^{Q(t)}\left(F_{u}\left(t, u_{n}\right), u_{n}-u\right) d t \\
& \quad \leq d_{2} \int_{0}^{T} c_{7} b(t)\left(u_{n}-u\right) d t \\
& \quad \leq c_{7} d_{2}\left\|u_{n}-u\right\|_{\infty} \int_{0}^{T} b(t) d t \rightarrow 0 .
\end{aligned}
$$

Combining (2.18) and (2.20), we can see that (2.15) holds. Therefore, by (2.15), $\Phi^{\prime}\left(u_{n}\right) \rightarrow 0$, $u_{n} \rightarrow u$ in $W$, and the definition of $\Phi^{\prime}$, we have

$$
\begin{aligned}
0 & =\lim _{n \rightarrow \infty}\left\langle\Phi^{\prime}\left(u_{n}\right), u_{n}-u\right\rangle \\
& =\lim _{n \rightarrow \infty}\left(u_{n}, u_{n}-u\right)-\lim _{n \rightarrow \infty} \int_{0}^{T} e^{Q(t)}\left(F_{u}\left(t, u_{n}\right), u_{n}-u\right) d t \\
& =\lim _{n \rightarrow \infty}\left\|u_{n}\right\|^{2}-\|u\|^{2}-0 .
\end{aligned}
$$

That is,

$$
\lim _{n \rightarrow \infty}\left\|u_{n}\right\|=\|u\|
$$

It follows from $u_{n} \rightarrow u$ in $W$ that

$$
\left\|u_{n}-u\right\|^{2}=\left(u_{n}-u, u_{n}-u\right) \rightarrow 0
$$

so $\left\{u_{n}\right\}$ has a convergent subsequence in $W$. Thus $\Phi$ satisfies the Cerami condition.

Since $\operatorname{dim} W^{0}$ and $\operatorname{dim} W^{-}$are finite, we can choose an orthonormal basis $\left\{e_{m}\right\}_{m=1}^{k_{1}}$ of $W^{0}$, an orthonormal basis $\left\{e_{m}\right\}_{m=k_{1}+1}^{k_{2}}$ of $W^{-}$, and an orthonormal basis $\left\{e_{m}\right\}_{m=k_{2}+1}^{\infty}$ of $W^{+}$, where $1 \leq k_{1}<k_{2}$ and $k_{1}+1 \leq k_{2}<\infty$. Then $\left\{e_{m}\right\}_{m=1}^{\infty}$ is an orthonormal basis of $W$. Let $X_{m}:=\mathbb{R} e_{m}$, then $Y_{k}$ and $Z_{k}$ can be defined as (2.3).

Lemma 2.4 If $Z_{k}=\overline{\bigoplus_{m \geq k} X_{m}}$, then

$$
\beta_{k}:=\sup _{u \in Z_{k},\|u\|=1}\|u\|_{\infty} \rightarrow 0 \quad \text { as } k \rightarrow \infty
$$

Proof It is clear that $0<\beta_{k+1} \leq \beta_{k}$, so $\beta_{k} \rightarrow \beta \geq 0, k \rightarrow \infty$. For every $k \in N$, there exists $u_{k} \in Z_{k}$ such that $\left\|u_{k}\right\|=1$ and $\left\|u_{k}\right\|_{\infty}>\frac{1}{2} \beta_{k}$. By the definition of $Z_{k}, u_{k} \rightarrow 0$ in $W$, then by Lemma 2.1 in [3] and Rellich's embedding theorem (see [5]), $u_{k} \rightarrow 0$ in $Z_{k}$. Therefore, we have $\beta=0$, that is, $\beta_{k} \rightarrow 0$.

Proof of Theorem 1.1 For the Hilbert space $W$, define $Y_{k}$ and $Z_{k}$ as in (2.3). According to Lemma 2.3 and the evenness of $F(t, \cdot)$, we know that $\Phi$ satisfies the Cerami condition $(C)$ and $\Phi(-u)=\Phi(u)$. It remains to verify conditions (i) and (ii) of Lemma 2.2. 
Verification of (i). Taking $r_{k}=\beta_{k}^{-1}$, using Lemma 2.4, one has that

$$
r_{k} \rightarrow+\infty \quad \text { as } k \rightarrow \infty
$$

Choose $k$ large enough such that $Z_{k} \subset W^{+}$and

$$
r_{k} \geq\left(4 \max _{s \in[0,1]} a(s) \int_{0}^{T} e^{Q(t)} b(t) d t\right)^{1 / 2} .
$$

Now, for $u \in Z_{k}$ with $\|u\|=r_{k}$ and $\left(F_{1}\right)$, we have that

$$
\begin{aligned}
\Phi(u) & =\frac{1}{2}\|u\|^{2}-\int_{0}^{T} e^{Q(t)} F(t, u) d t \\
& \geq \frac{1}{2}\|u\|^{2}-\max _{s \in\left[0,\|u\|_{\infty}\right]} a(s) \int_{0}^{T} e^{Q(t)} b(t) d t \\
& \geq \frac{1}{2}\|u\|^{2}-\max _{s \in\left[0, \beta_{k}\|u\|\right]} a(s) \int_{0}^{T} e^{Q(t)} b(t) d t \\
& \geq \frac{1}{2}\|u\|^{2}-\max _{s \in[0,1]} a(s) \int_{0}^{T} e^{Q(t)} b(t) d t \\
& \geq \frac{r_{k}^{2}}{4},
\end{aligned}
$$

which implies that

$$
a_{k}=\inf _{u \in Z_{k},\|u\|=r_{k}} \Phi(u) \geq \frac{r_{k}^{2}}{4} \rightarrow+\infty \quad \text { as } k \rightarrow \infty
$$

Verification of (ii). Similar to Lemma 2.3 in [2], we get that, for a finite-dimensional subspace $Y_{k} \subset W$, for any $k \in \mathbb{N}$, there exists a constant $\epsilon_{k}>0$ such that

$$
m\left(\Lambda_{u}^{k}\right) \geq \epsilon_{k}, \quad \forall u \in Y_{k} \backslash\{0\}
$$

where $m(\cdot)$ denotes the Lebesgue measure in $\mathbb{R}, \Lambda_{u}^{k}:=\left\{t \in[0, T]:|u| \geq \epsilon_{k}\|u\|\right\}$. Note that $e^{Q(t)} \geq d_{1}$ for some constant $d_{1}>0(\forall t \in[0, T])$. By $\left(F_{2}\right)$, for any $k \in \mathbb{N}$, there exists a constant $S_{k}>0$ such that

$$
F(t, u) \geq \frac{|u|^{2}}{d_{1} \epsilon_{k}^{3}}, \quad \forall t \in[0, T], \forall|u| \geq S_{k} .
$$

Hence, using (2.23), (2.24), $\left(F_{2}\right)$, and $e^{Q(t)} \geq d_{1}$, we have that, for any $k \in \mathbb{N}$ and $u \in Y_{k}$ with $\|u\| \geq S_{k} / \epsilon_{k}$

$$
\begin{aligned}
\Phi(u) & \leq \frac{1}{2}\left\|u^{+}\right\|^{2}-\int_{0}^{T} e^{Q(t)} F(t, u) d t \\
& \leq \frac{1}{2}\|u\|^{2}-\left(\int_{[0, T] \backslash \Lambda_{u}^{k}} e^{Q(t)} F(t, u) d t+\int_{\Lambda_{u}^{k}} e^{Q(t)} F(t, u) d t\right) \\
& \leq \frac{1}{2}\|u\|^{2}-\int_{\Lambda_{u}^{k}} e^{Q(t)} F(t, u) d t
\end{aligned}
$$




$$
\begin{aligned}
& \leq \frac{1}{2}\|u\|^{2}-d_{1} \int_{\Lambda_{u}^{k}} \frac{|u|^{2}}{d_{1} \epsilon_{k}^{3}} d t \\
& \leq \frac{1}{2}\|u\|^{2}-\frac{d_{1} \epsilon_{k}^{2}\|u\|^{2}}{d_{1} \epsilon_{k}^{3}} m\left(\Lambda_{u}^{k}\right) \\
& \leq \frac{1}{2}\|u\|^{2}-\|u\|^{2} \\
& =-\frac{1}{2}\|u\|^{2} .
\end{aligned}
$$

Now, for any $k \in \mathbb{N}$, if we choose

$$
\|u\|=\rho_{k}>\max \left\{\gamma_{k}, S_{k} / \epsilon_{k}\right\}
$$

then (2.25) implies that

$$
b_{k=} \max _{u \in Y_{k},\|u\|=\rho_{k}} \Phi(u) \leq-\frac{1}{2} \rho_{k}^{2} \leq 0, \quad \forall k \in \mathbb{N}
$$

Consequently, by Lemma 2.2, system (1.1) has infinitely many nontrivial $T$-periodic solutions.

\section{Conclusion}

We obtain infinitely many nontrivial periodic solutions for a class of damped vibration systems with superquadratic terms at infinity. By using some weaker conditions, our results extend and improve some existing results in the literature.

\section{Funding}

Research supported by the National Natural Science Foundation of China (No. 11771182) and the Natural Science Foundation of Shandong Province (No. ZR2017JL005).

\section{Abbreviations}

Not applicable.

Availability of data and materials

Not applicable.

Ethics approval and consent to participate

Not applicable.

Competing interests

The authors declare that they have no competing interests.

\section{Consent for publication}

Not applicable.

Authors' contributions

All authors contributed equally to the writing of this paper. All authors read and approved the final manuscript.

\section{Publisher's Note}

Springer Nature remains neutral with regard to jurisdictional claims in published maps and institutional affiliations.

Received: 18 September 2019 Accepted: 9 December 2019 Published online: 13 December 2019

\section{References}

1. Bartsch, T.: Infinitely many solutions of a symmetric Dirichlet problem. Nonlinear Anal. TMA 20, 1205-1216 (1993)

2. Chen, G.: Infinitely many nontrivial periodic solutions for damped vibration problems with asymptotically linear terms. Appl. Math. Comput. 245, 438-446 (2014) 
3. Chen, G.: Periodic solutions of superquadratic damped vibration problems. Appl. Math. Comput. 270, 794-801 (2015)

4. Li, X., Wu, X., Wu, K.: On a class of damped vibration problems with super-quadratic potentials. Nonlinear Anal. 72, $135-142(2010)$

5. Willem, M.: Minimax Theorems. Birkhäuser, Boston (1996)

6. Wu, X., Chen, S., Teng, K.: On variational methods for a class of damped vibration problems. Nonlinear Anal. 68, 1432-1441 (2008)

7. Zou, W.: Variant fountain theorems and their applications. Manuscr. Math. 104, 343-358 (2001)

Submit your manuscript to a SpringerOpen ${ }^{\circ}$ journal and benefit from:

- Convenient online submission

- Rigorous peer review

Open access: articles freely available online

- High visibility within the field

- Retaining the copyright to your article

Submit your next manuscript at $\gg$ springeropen.com 